\title{
Removal of baseline noise from Electrocardiography (ECG) signal based on time domain approach
}

\author{
Duong Trong Luong, Nguyen Duc Thuan, Trinh Quang Duc
}

Dept. of Electronics Technology and Biomedical Engineering, Hanoi University of Science and Technology, Hanoi, Vietnam

\section{Email address:}

luong.duongtrong@hust.edu.vn(D. T. Luong), thuan.nguyenduc@hust.edu.vn (N. D. Thuan), duc.trinhquang@hust.edu.vn (T. Q. Duc)

To cite this article:

Duong Trong Luong, Nguyen Duc Thuan, Trinh Quang Duc. Removal of Baseline Noise from Electrocardiography (ECG) Signal Based on time Domain Approach. International Journal of Biomedical Science and Engineering. Vol. 2, No. 2, 2014, pp. 11-16.

doi: $10.11648 / j . i j b s e .20140202 .11$

\begin{abstract}
Electrocardiographic (ECG) signals in measurements are often contaminated with different types of noises in which include baseline noise. In case of the frequency of baseline noise is greater or smaller than frequency of the ECG signal, it is easy to filter the baseline noise from ECG signal by using filtered methods in frequency domain. In contrast, if frequency of baseline noise and frequency of ECG signal are coincident, it is difficult to apply the frequency domain filters for baseline noise removal. In this paper, we introduce an approach to remove of baseline noise from ECG signal in time domain and evaluation the efficacy of the method based on Mean Square Error criteria. We have performed experiments with simulated ECG signal which including white noise, random and sinusoidal baseline noises. Throughout the experiment, we found that the errors of time domain filters depend on the amplitude of the base line noises.
\end{abstract}

Keywords: Time Domain, ECG Signal Processing, Baseline Noise, Simulated ECG Signal

\section{Introduction}

The baseline noise from ECG signal measurement is caused by fluctuations of the impedance between electrodes and skin; patient's movements and respiration [1]. The baseline noise also called wandering baseline, generated randomly with low frequency and very hard to analyze since their amplitudes and frequencies are not stationary [2]. Normally, baseline noise is a low frequency additive noise over range $0 \div 0.8 \mathrm{~Hz}$, particularly overlaps the band of ECG signal [3]. Sometime, the frequency of baseline noise may be greater than $1 \mathrm{~Hz}$. The in-band frequency of this type of noise makes its removal difficult without affecting the ECG [1]. To remove the baseline noise a number of studies had been proposed such as using high-pass filtering with cutoff frequency of about $0.8 \mathrm{~Hz}$ [5]. However, this is not always available, since it introduces distortions in the ECG signal, particularly in the ST segment. Adaptive filtering techniques have been developed and applied for ECG signals [6]. But in some cases, these techniques show the difficulty in obtaining a suitable reference signal, limiting the wide application of the method. Wavelet transform methods also used for removing baseline noise involve wavelet threshold [7-8-9], discrete wavelet transform DWT [10], wavelet packet WP [11]. Although these methods are promising but they remain have disadvantage that the precision of the wavelet filters depends on the range of the frequency variation as well as amplitude of the baseline wandering and it strongly varied when the frequency of the noise reaches to the threshold frequency [2]. Generally, it is easy to filter the baseline noise from ECG signal by using filtered methods in frequency domain in case of baseline noise frequency is greater or smaller than ECG signal frequency. But, it isn't effective for using these filtered methods if frequency of baseline noise and frequency of ECG signal are coincident. This paper, we introduce a new approach to remove of baseline noise from ECG signal in time domain. This method based on the concept of searching all of the points are zero aberration and shifting the points go to the zero value which are original values of these points. From the zero aberration points, a curve was interpolated to estimate the baseline noise. The error of this method is error of the interpolated curve compared with the baseline noise curve. The points that their original values are zero called isoelectric points or silent points. These points will be determined based on their specific mathematical characteristics. To interpolate the curve, the technique termed as Cubic spline interpolation is applied to estimate the curve with third order line then the zero aberration points returned to zero by subtraction of the ECG signal to the interpolated curve [12]. To estimate the 
error of this method, this work uses Mean Square Error (MSE) criteria.

\section{Materials and Method}

To carry out the method for determination of the isoelectronic points experimentally, a simulated clean ECG signal is generatedwith basically several parameters such as heart rate is $72 \mathrm{bpm}$ (frequency equivalence is $1.2 \mathrm{~Hz}$ ); 10 QRS peaks, sampling rate is $360 \mathrm{~Hz}$; the length of interval is $10 \mathrm{~s}$; amplitude is $1,6 \mathrm{mV} \quad(1,2 \mathrm{mV}$ : upper limit; $-0,4 \mathrm{mV}$ :lower limit) as shown in the Fig.1.a. The positions of silent points are shown in Fig.1.b. These points termed as P_onset (begin of P wave), QRS_onset (begin of QRS complex wave), QRS_offset (end of QRS complex wave) and T_offset (end of T wave). QRS_onset and QRS_offset are inflexion points, their mathematical characteristic are zero crossing points of second derivation. Similarly to QSR_offset and QRS_onset, T_offsets and P_onset are also inflexion, since it changes the signal state of $\overline{\mathrm{P}}$ and $\mathrm{T}$ waves to the silent state, thus the points are also zero crossing points of second derivation.
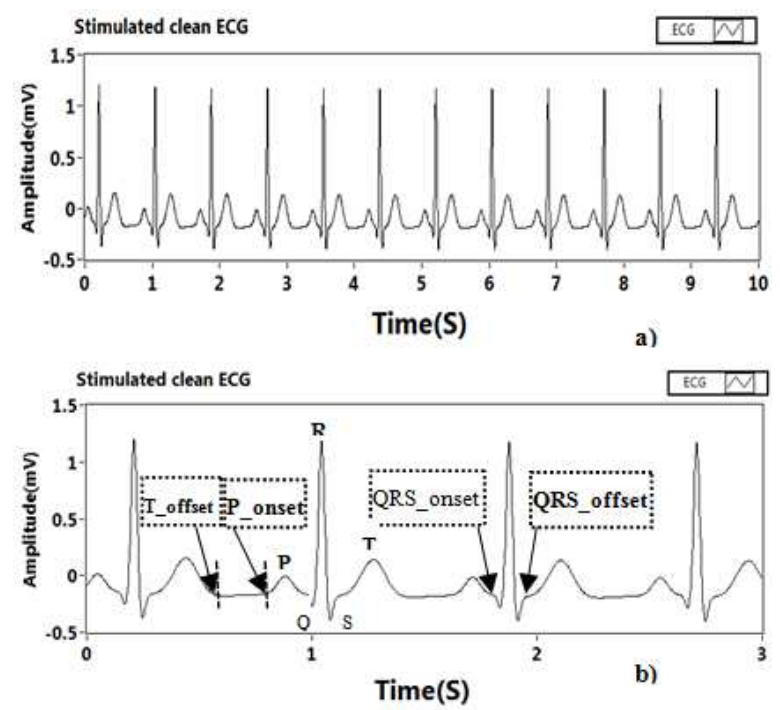

Figure 1. a) The simulated clean ECG signal. b) silent points position.

Aspect of biology, these points correspond with beginning and the end of period of heart beat, in this case generated potential deviation is zero. When values of the silent points are different to zero, this indicates that the baseline noise is generated. Removing of baseline noise is the shifting silent points return to zero value. When the locations of the silent points are determined, Cubic spline technique estimates a continuous baseline by joining silent points with third order line (spline). Thus the efficacy of this technique depends on the accuracy of the silent point detection as well as interpolation. One of the reasons effects of the accuracy of the silent point detection is white noise.
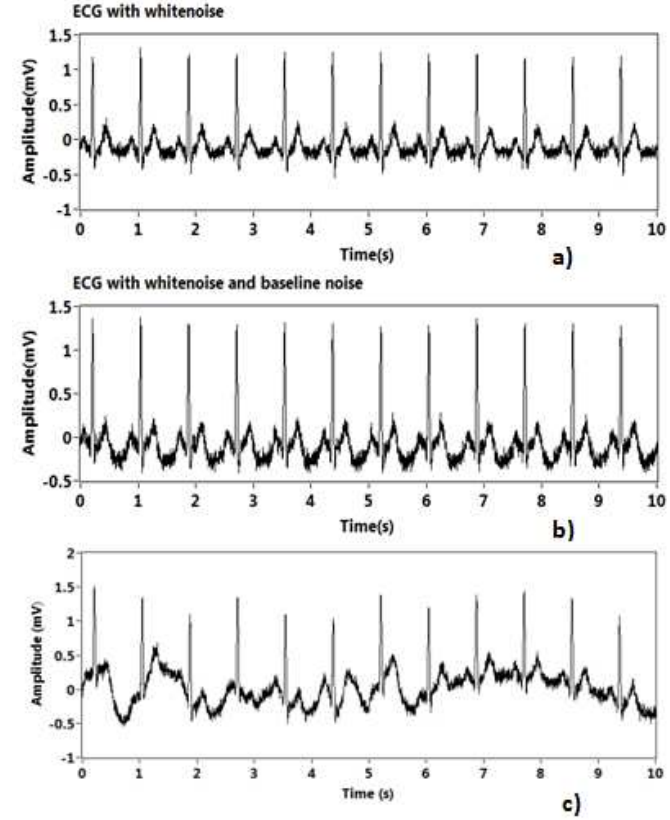

Figure 2. a) The simulated clean ECG signal superposed with white noise. b) The simulated clean ECG signal superposed with white noise and sinusoidal baseline noise with variation amplitudes from 0.01 to 1milivolt and frequency coincides with frequency of simulated ECG signal. C) The simulated clean ECG signal superposed with white noise and random baseline noise with variation amplitude from 0.01 to 0.5 milivolt and frequency coincides with frequency of simulated ECG signal.

To simulate the measured ECG signal practically and examine the capabilities of the time domain filter method, we used the simulated clean ECG signal is superposed with white noise as shown in Fig.2.a. For simplicity, the baseline noise can be considered as sinusoidal waves and the relationship between MSE and amplitude of the baseline noise can be investigated with variation amplitudes from 0.01 to 1 milivolt and frequency coincides with frequency of simulated clean ECG signal as shown in Fig.2.b.

To evaluate the time domain filter in assumption of measurement conditions, we also used thesimulated clean ECG signal is superposed with white noiseand random baseline noise with variation amplitude from 0.01 to 0.5 milivolt and frequency coincides with frequency of simulated clean ECG signal as shown in Fig.2.c

If the simulated clean ECG signal is $X(t)$, the baseline noise is $B(t)$ and the white noise is $W(t)$, the input ECG signal to experiment can be simply expressed as:

$$
S(t)=X(t)+B(t)+W(t)
$$

The curve B'(t) interpolated by Cubic spline algorithm can be assigned as the baseline noise waves and with the average filter the white noise $\mathrm{W}(\mathrm{t})$ is reduced and neglected. Therefore, by the subtraction of $S(t)$ - B'(t), we can obtain the nearly clean ECG signal.

\section{Implementation}

As mentioned above, white noise affects the accuracy of the detection of silent points significantly. Hence, white 
noise level reduction is necessary and smoothened by the average filter before the silent point detection. However, smooth signal processing is able to affect locations of silent points. So, the first detection of $\mathrm{P}, \mathrm{QRS}$ and $\mathrm{T}$ peaks are performed then silent points are detected respectively. Detection of these points can be carried out as following four steps as well as the flow chart show as in the fig.3

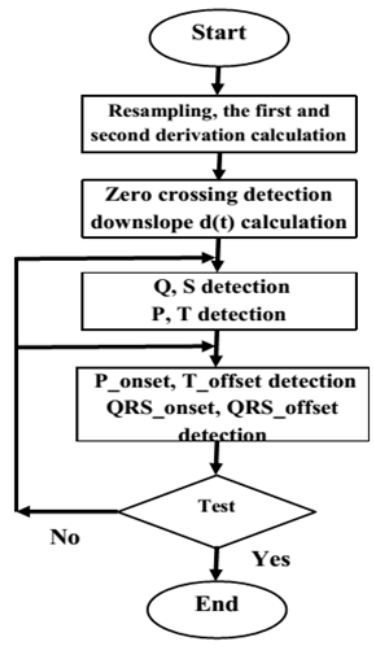

Figure 3. Silent points detection flow chart

1) Resampling and smoothing signal $S(t)$, calculating the first and second derivation. These processes implemented to find out a set of characteristics points (maximum and minimum points) and reduce the influence of white noise signal $\mathrm{W}(\mathrm{t})$ simultaneously. Determining crossing points from the first and second derivation calculations. This is the basic determination of the silent points in steps 2 and 3 . Based on the calculation of the first derivation, we can also determine the location of reached extreme slopes before and after the $\mathrm{R}$ peak. We used a loop for testing relative positions between slopes to check if it is a negative slope or positive slope of the QRS waves.

2) Detecting the $P, T, Q$, and $S$ peaks from crossing points of the first derivations. To estimate the error of location of detected points with influence of white noise, baseline noise and smoothened signal $\mathrm{S}(\mathrm{t})$, real positions of points of the clean ECG signal are compared with detected positions of those points in case of the clean ECG signal superposed with white noise and baseline noise. For this purpose, we use Mean Square Error (MSE) criteria which presented by formula (2)

$$
\operatorname{MSE}=\frac{1}{N} \sum_{i=1}^{N}\left(\widehat{x}_{\imath}-x_{i}\right)^{2}
$$

where $\mathrm{N}$ is the number of elements of ECG signal value array to calculate MSE, $x_{i}$ and $\widehat{x}_{\boldsymbol{\imath}}$ are real positions of the clean ECG signal and detected positions of those points in case of white noise and baseline noise at the $i^{\text {th }}$ position, respectively.

3) Detect $P$ _onset and $T$ _offset points from crossing points of the first derivation; detect $\mathrm{P}_{-}$onset and $\mathrm{T}$ _offset points from crossing points of the second derivation (inflexion points) respectively. Finally, based on calculating the second derivation, QRS_onset and QRS_offset points are detected from crossing point.

4) Performing test to remove points which aren't necessary determined points. These points are generated by affecting of white noise. The loop is carried out to detect all of points that begin and end of component waves in the $S(t)$ signal for interpolation of baseline noise then removed this baseline noise by shifting silent points return to zero with a subtraction of the noised ECG to the interpolated curve. This algorithm is installed in Labview 2012 to perform the series of numerical experiments.

\section{Results and Discussions}

To examine the time domain filter algorithm, we carried out the experiment with the conditions of simulated clean ECG signal has frequency is $1.2 \mathrm{~Hz}$. This signal is superposed with sinusoidal baselines noise with variant amplitude from 0.01 to 1 milivolt, its frequency coincides with frequency of simulated clean ECG signal (as show in the fig.4); the white noise with 0.02 in amplitude is superposed.

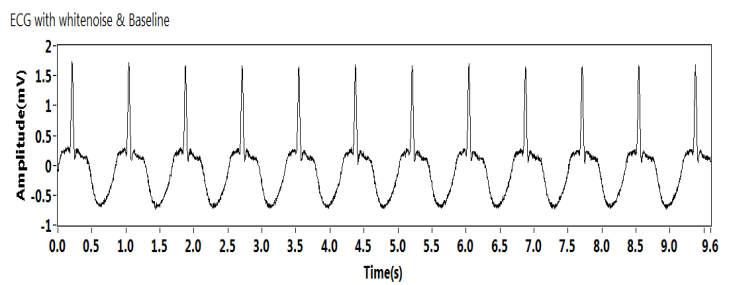

Figure 4. The input ECG signal for applying of the purposed method.

To investigate the relationship between MSE and amplitude of the baseline noise, MSE values were collected and presented in graphs. The filter algorithm was examined with each feature as well as combination of them sequentially. Figure 5, 6, 7 and 8 show the MSE of location of QRS_onset points; QRS_onset and QRS_offset points; QRS_onset and QRS_offset and T_offset points; QRS_onset and QRS_offset and T_offset and $P_{-}$onset points respectively between the simulated clean ECG with the simulated clean ECG signal is superposed with white noise and sinusoidal baseline noise with variation magnitude from 0.01 to 1 milivolt and frequency coincides with frequency of simulated ECG signal.

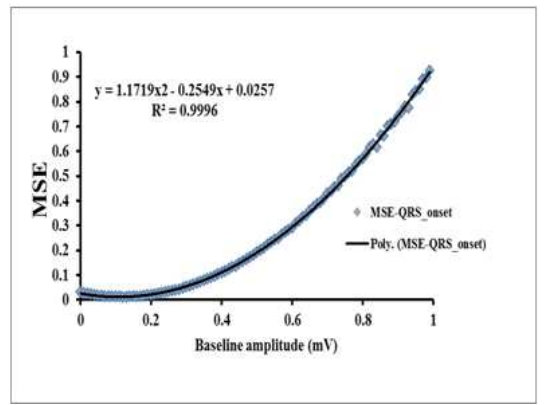

Figure 5. MSE values and polynomial of set of QRS_onset points. 


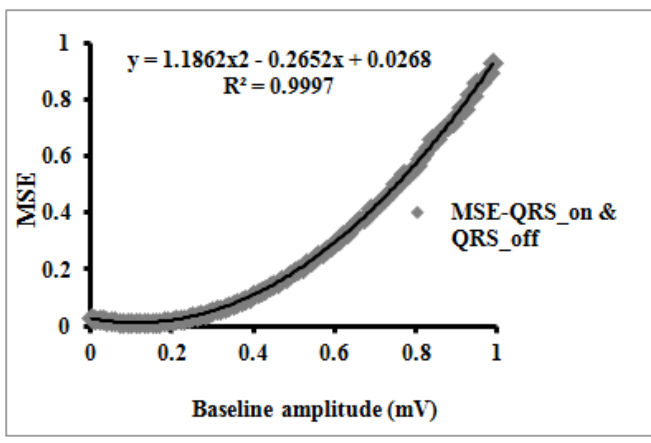

Figure 6. MSE values and polynomial of set of QRS_onset and QRS_offset points.

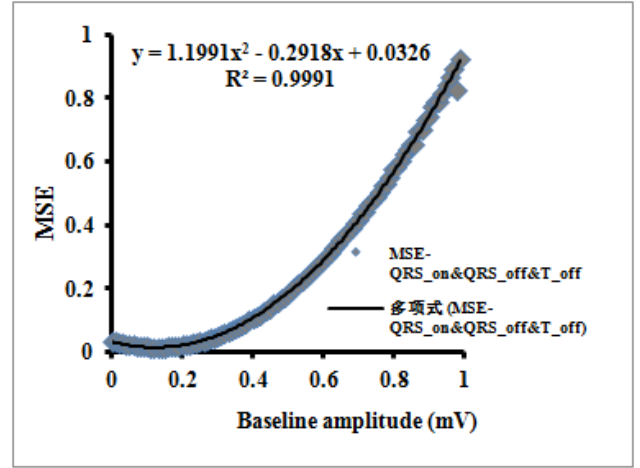

Figure 7. MSE values and polynomial of set of QRS_onset and QRS_offset and T_offset points.

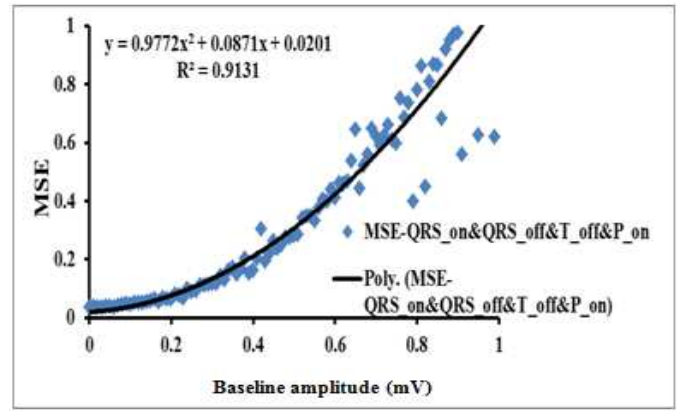

Figure 8. MSE values and polynomial of set of QRS_onset and QRS_offset and T_offset and P_onset points.

As the figures shown, the MSE compared between the interpolated curve with the silent points or in other words the MSE compared between noised ECG after filtered and the clean ECG signal is quadratic proportional to the amplitude of the baseline noise. This means the error of the filtered ECG signal is proportional to the amplitude of the baseline noise, because the MSE is presented in quadratic the function expressed in Eq.2. Although white noise reducing is performed before detecting of silent points but from results of figure 5, 6, 7 and 8 show that the MSE in the case of use silent points of QRS_onset and QRS_offset points seems fitted to regression function with higher accuracy than the others. After carrying out the experiment in the conditions of QRS_onset, QRS_offset and T_offset, we obtained the result as shown in Fig.9. As the figure shown, the baseline noise is reduced from the ECG signal apparently.

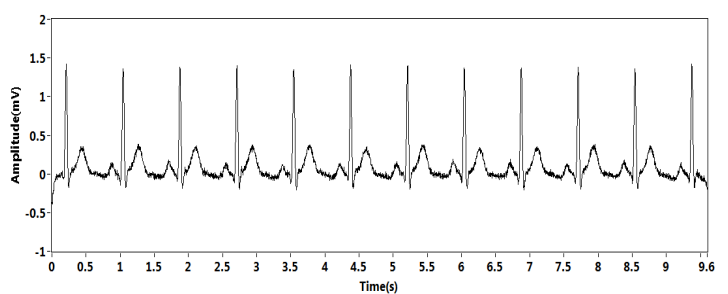

Figure 9. The ECG signal after removing the baseline noise.

Typically, the baseline noise is not uniform sinusoidal waves but random amplitude. Therefore, we examined the filter algorithm with random baseline noise amplitude. Figure 10,11, 12 and 13 show the error of location of QRS_onset points; QRS_onset and QRS_offset points; QRS_onset and QRS_offset and T_offset points; QRS_onset and QRS_offset and T_offset and P_onset points respectively between the simulated clean ECG with the simulated clean ECG signal is superposed with white noise and random baseline noise with variation amplitude from 0.01 to 0.5 milivolt and frequency coincides with frequency of simulated ECG signal.

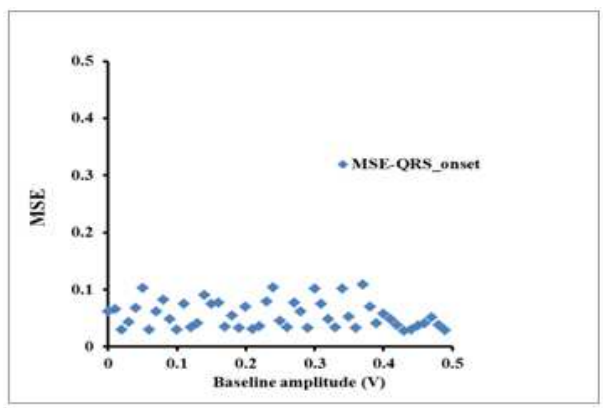

Figure 10. MSE values of set of QRS_onset points.

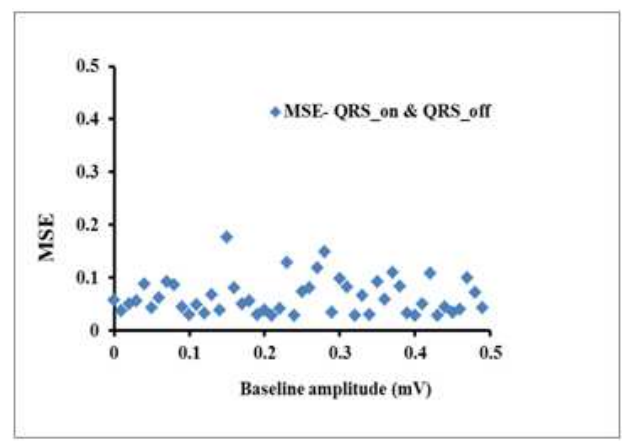

Figure 11. MSE values of set of $Q R S \_$onset and $Q R S \_$offset points.

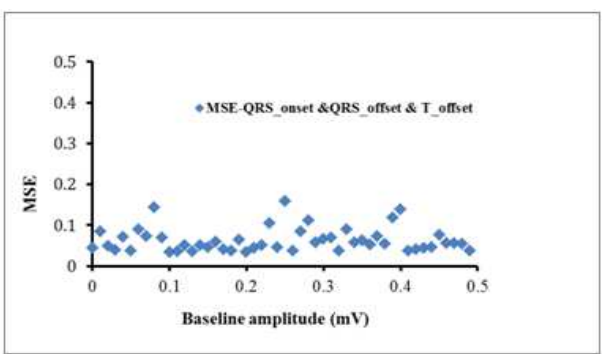

Figure 12. MSE values of set of QRS_onset and QRS_offset and T_offset points. 


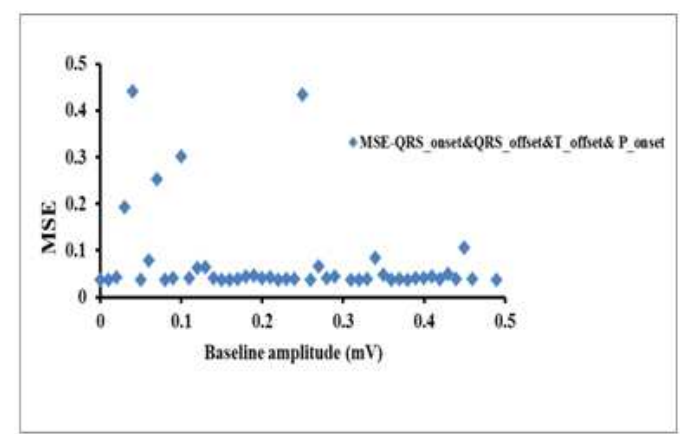

Figure 13. MSE values of set of QRS_onset and QRS_offset and T_offset and P_onset points.

Similar to the Fig.8, the result presented in the Fig.13 shows that detection of silent points when added $\mathrm{P}_{-}$onset point is less accurate. This represents the difficulty to detect $\mathrm{P}$ _onset points while white noise and random baseline noise are superposed with the ECG signal specially. We also experimented the purposed method with the simulated clean ECG signal has frequency is $1.2 \mathrm{~Hz}$ and superposed with random baselines noise with fluctuation magnitude up to 0.5 milivolt, its frequency coincides with frequency of simulated clean ECG signal as show in the fig. 14 .

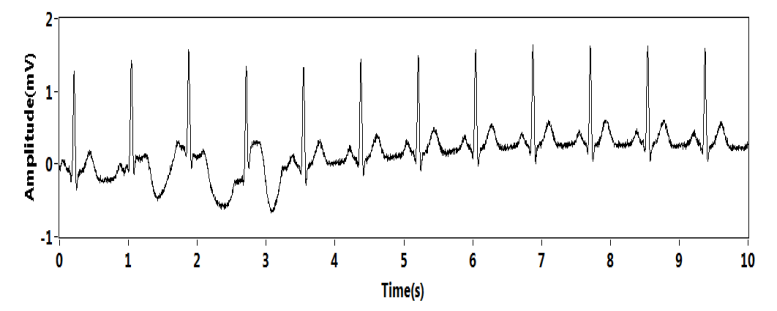

Figure 14. The simulated clean ECG signal is superposed with random baseline noise

After applying the time domain filter algorithm with the features of QRS_onset, QRS_offset and T_offset for the random amplitude baseline noise circumstances, the baseline noise has reduced from the ECG signal as shown in the fig. 15 .

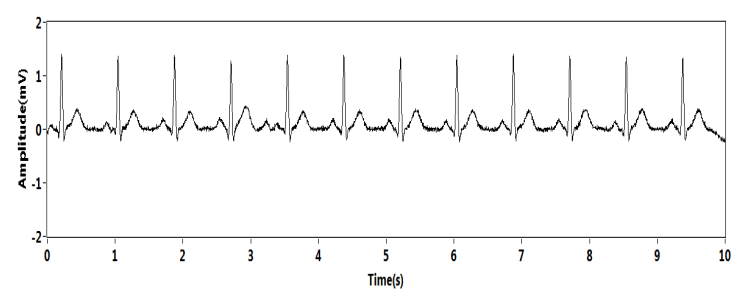

Figure 15. The ECG signal after removing the random baseline noise.

\section{Conclusion}

The approach to remove of baseline noise from ECG signal in time domain was investigated with application of the definition and determination of the isoelectronic points (silent points) based on their mathematical characteristics as well as calculation of the first and second derivation. The loop is applied to detect all of silent points for interpolating of baseline noise and then removing of this baseline noise by shifting silent points return to zero. The purposed method was experimented with two types of baseline noise with variant amplitude up to 1 milivoltage and frequency coincides with frequency of the ECG signal. The efficacy of this method depends on the accuracy of the silent point detection and interpolation. This is estimated by using MSE criteria. With the detection of isoelectronic points such as QRS_onset; QRS_onset \& QRS_offset; QRS_onset \& QRS_offset \& T_offset, the fluctuation of the error after baseline noise filtered is low. The accuracy decreasing of the regressive function after added $P_{-}$onset points shows the high error fluctuation and less reliability. Although the filtered algorithm was experimented with a single loop, this paper has demonstrated the potential algorithm to remove the baseline noise with in-band frequency. In the near future, the algorithm with multi-loops of this filter will be used toimprove the accuracy of the in-band frequency baseline noise removal.

\section{References}

[1] A. Fasano, V. Villani, L. Vollero "Baseline Wander Estimation and Removal by Quadratic Variation Reduction". The 33rd Annual International Conference of the IEEE EMBS, USA, August 30 - September 3, 2011.

[2] Duong Trong Luong, Nguyen Duc Thuan, Chu Duc Hoang, Nguyen Van Trang, Trinh Quang Duc "Study on limitation of removal of baseline noise from electrocardiography signal in measurement using wavelet analysis “. The $5^{\text {th }}$ International Conference on Ubiquitous and Future Networks (ICUFN) of the IEEE Xplore. ISSN 2165-8528; page(s): 481-486; Da Nang, VietNam; 2-5 July 2013.

[3] L. T. Sheffield, C. A. Berson, D. B. Remschel, P. C. Gillette, R. E. Hermes, L. Hinkle, H. Kennedy, D. M. Mirvis, and C. Oliver, "Recommendations for standard of instrumentation and practice in the use of ambulatory electrocardiography," Circulation, pp. 626A-636A, 1985.

[4] Yan Sun, Kap Luk Chan, Shankar Muthu Krishnan, "ECG signal conditioning by morphological filltering". Computers in Biology and Medicine 32 (2002) 465-479, 0010-4825/2002 Elsevier Science Ltd.All rights reserved.

[5] J. A. Van Alste and T. S. Schilder, "Removal of base-line wander and power-line interference from the ECG by an efficient FIR filter with a reduced number of taps," IEEE Transactions on Biomedical Engineering, vol. 32, no. 12, pp. 1052-1060, Dec 1985.

[6] Jungkuk Kim, Minkyu Kim, Injae Won, Seungyhul Yang, Kiyoung Lee, and Woong Huh, “ An ECG signal processing Algorithm based on removal of wave deflections in time domain". The 31st Annual International Conference of the IEEE EMBS, Minneapolis, Minnesota, USA, September 2-6, 2009.

[7] G.Umamaheswara Reddy, M. Muralidhar, Dr. S. Varadarajan "ECG De-Noising using improved thresholding based on Wavelet transforms", International Journal of Computer Science and Network Security, 9(9), 221-225, 2009. 
[8] Mikhled Alfaouri and Khaled Daqrouq, "ECG Signal Denoising By Wavelet Transform Thresholding". American Journal of Applied Sciences 5 (3), 276-281, 2008.

[9] P. Karthikeyan, M. Murugappan, and S.Yaacob, "ECG Signal Denoising Using Wavelet Thresholding Techniques in Human Stress Assessment", International Journal on Electrical Engineering and Informatics 4 (2), 306-319, 2012.

[10] Chissanuthat Bunluechokchai and Theera Leeudomwong "Discrete Wavelet Transform-based Baseline Wandering Removal for High Resolution Electrocardiogram". International Journal of applied biomedical engineering, 2010 .
[11] Behzad Mozaffary Mohammad A. Tinati, "ECG Baseline Wander Elimination using Wavelet Packets". World Academy of Science, Engineering and Technology 3, 550-552, 2007.

[12] Fabio Badilini, Arthur J. Moss, Edward L. Titlebaum "Cubic spline baseline estimation in ambulatory ECG recordings for the measurement of ST segment displacements", Annual Internatlonal Conference of the IEEE Engineering in Medicine and Biology Society, Vol. 13. No. 2, 1991. 